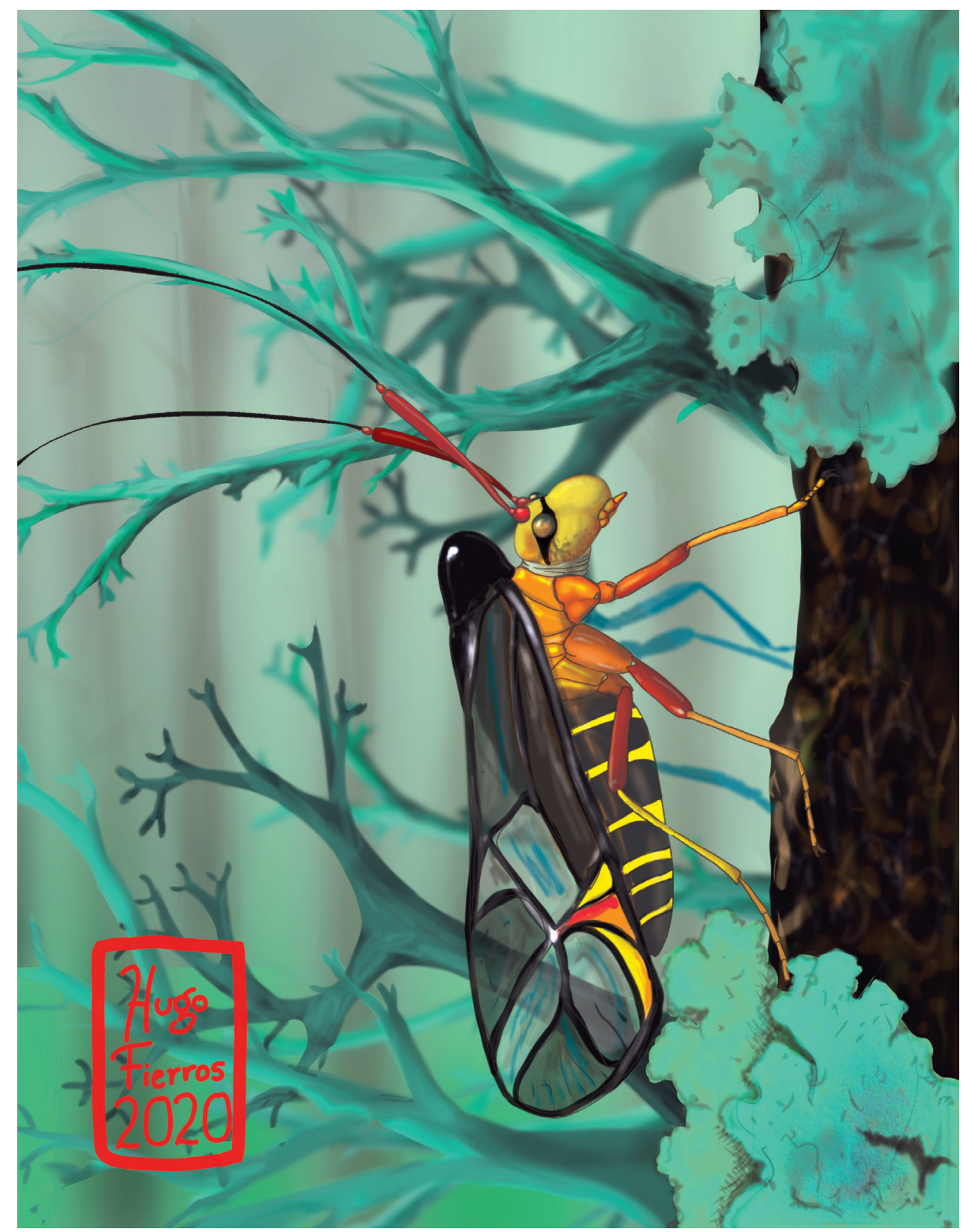

Dugesiana, Año 27, No. 2, julio 2020-diciembre 2020 segundo semestre de 2020), es una publicación semestral, editada por la Universidad de Guadalajara, a través del Centro de Estudios en Zoología, por el Centro Universitario de Ciencias Biológicas y Agropecuarias. Camino Ramón Padilla Sánchez \# 2100, Nextipac, Zapopan, Jalisco, Tel. 37771150 ext. 33218, http://148.202.248.171/dugesiana/index.php/DUG/index, glenusmx@gmail.com. Editor responsable: José Luis Navarrete-Heredia. Reserva de Derechos al Uso Exclusivo 04-2009-062310115100203, ISSN: 2007-9133, otorgados por el Instituto Nacional del Derecho de Autor. Responsable de la última actualización de este número: José Luis Navarrete-Heredia, Editor y Ana Laura González-Hernández, Asistente Editorial. Fecha de la última modificación 1 de julio 2020, con un tiraje de un ejemplar.

Las opiniones expresadas por los autores no necesariamente reflejan la postura del editor de la publicación.

Queda estrictamente prohibida la reproducción total o parcial de los contenidos e imágenes de la publicación sin previa autorización de la Universidad de Guadalajara. 
Artículo

\title{
Scolytinae y Platypodinae (Coleoptera: Curculionidae) de un bosque mixto de pino-latifoliadas de la Sierra de Manantlán, Jalisco, México
}

\author{
Scolytinae y Platypodinae (Coleoptera: Curculionidae) from mixed pine-latifoliated forest of \\ the Sierra de Manantlán, Jalisco, Mexico
}

\author{
Aranzazú E. Castorena Pérez ${ }^{1 *}$; Armando Equihua Martínez²; Enrique J. Jardel Peláez ${ }^{3}$; Luis Euge- \\ nio Rivera Cervantes ${ }^{3}$; y Ramón Cuevas Guzmán ${ }^{3}$ \\ ${ }^{*}$ Maestría en Ciencias en Manejo de Recursos Naturales, Centro Universitario de la Costa Sur, Universidad de \\ Guadalajara. aranzazucastorena@gmail.com; ${ }^{2}$ Laboratorio de Entomología Forestal, Instituto de Fitosanidad, Co- \\ legio de Postgraduados, Montecillo, Km 36.5 Carretera Méx-Texcoco; Montecillo Texcoco, Estado de México, \\ C.P. $56230 ;{ }^{3}$ Departamento de Ecología y Recursos Naturales, Centro Universitario de la Costa Sur, Universidad \\ de Guadalajara. Av. Independencia Nacional 151, Autlán de Navarro, Jal. C.P. 48900.
}

\begin{abstract}
RESUMEN
Como parte de una evaluación de causas de mortalidad de árboles, se determinó la composición de especies de escarabajos de la corteza en un bosque mixto de pino-latifoliadas en la Reserva de la Biosfera Sierra de Manantlán, Jalisco, México. En dos rodales con condiciones ambientales similares se realizaron colectas mensuales utilizando dos tipos de trampas (recipientes con alcohol al 70\% y láminas de pegamento; 46 de cada tipo), cubriendo un ciclo anual (julio de 2018 - agosto de 2019). Fueron colectadas y determinadas 17 especies de la familia Curculionidae (15 Scolytinae y dos Platypodinae); adicionalmente, se registraron siete taxones de las familias Histeridae (tres), Cleridae (tres) y Trogossitidae (una) que se comportan como depredadores de escarabajos de la corteza. Diez de las especies identificadas son nuevos registros para el estado de Jalisco: Amphicranus rameus, A. melanurus, Corthylus comatus, C. fuscus, Gnathotrichus sulcatus, Hylurgops incomptus, Monarthrum laterale, Pseudips mexicanus (Scolytinae) y Euplatypus longius y E. pini (Platypodinae). Los rodales mostraron diferencias en la abundancia de individuos colectados, lo cual se atribuye a la fase de desarrollo del brote de las poblaciones de descortezadores al momento de la medición. Los escarabajos ambrosiales fueron los más abundantes, seguidos por los Histeridae, Dendroctonus, Cleridae y Trogossitidae. La comparación del número de capturas por tipo de trampa sólo mostró diferencias significativas para los ambrosiales $(\mathrm{t}=3.53, \mathrm{p}=0.001)$, siendo más efectivas las trampas de alcohol que las de pegamento (media del número de capturas $19.7 \pm 2.3$ y $8.8 \pm 2$ respectivamente). La mayor parte de las especies encontradas tienen amplia distribución geográfica, pero no se habían registrado para el estado Jalisco, lo cual pone de manifiesto la necesidad de más estudios taxonómicos, ecológicos y biogeográficos sobre los escarabajos de la corteza, teniendo en consideración su importancia ecológica y económica en los ecosistemas forestales.

Palabras clave: Dendroctonus, escarabajos ambrosiales, Estación Científica Las Joyas, mortalidad de árboles, Pinus douglasiana, escarabajos de importancia forestal.
\end{abstract}

\begin{abstract}
The composition of bark beetle species in a mixed pine-broadleafed forest was determined, as part of an evaluation of tree mortality at the Sierra de Manantlán Biosphere Reserve, Jalisco, Mexico. In two stands with the same environmental conditions, monthly collections were made using two types of traps (containers with $70 \%$ alcohol and glue sheets; 46 traps of each type), covering an annual cycle (July 2018 - August 2019). Seventeen species of the Curculionidae family (15 Scolytinae and two Platypodinae) were collected and determined; additionally, seven taxa were registered that behave like predators of bark beetles, of the families Histeridae (three), Cleridae (three) and Trogossitidae (one). Ten of the species identified are new records for the state of Jalisco: Amphicranus rameus, A. melanuros, Corthylus comatus, C. fuscus, Gnathotrichus sulcatus, Hylurgops incomptus, Monarthrum laterale, Pseudips mexicanus (Scolitynae) y Euplatypus longius, and E. pini (Platypodinae). The stands showed differences in the abundance of individuals collected, which is attributed to the bark beetles outbreak development phase. Ambrosial beetles were the most abundant, followed by the Histeridae, Dendroctonus, Cleridae and Trogossitidae. The comparison of the number of catches by trap type only showed significant differences for the ambrosial beetles $(\mathrm{t}=3.53, \mathrm{p}=0.001)$, those of alcohol being more effective than glue sheets (average number of captures $19.7 \pm 2.3$ and $8.8 \pm 2$ respectively). Most of the species found have a wide geographical distribution, but they had not been registered for the state of Jalisco, which highlights the need for more taxonomic, ecological and biogeographical studies on bark beetles, taking into account their ecological and economic importance in forest ecosystems.
\end{abstract} tality.

Key words: ambrosial beetles, Dendroctonus, forest importance beetles, Las Joyas Research Station, Pinus douglasiana, tree mor- 
Las subfamilias Scolytinae y Platypodinae (Coleoptera: Curculionidae), constituyen un grupo grande y diverso de especies de escarabajos barrenadores de la corteza de árboles (Atkinson 2017). Son un componente importante de los ecosistemas forestales porque los brotes de las poblaciones de estos insectos influyen en la composición, estructura y dinámica de los bosques y pueden ser causa de la muerte masiva de árboles (Equihua y Burgos 2002; Burgos-Solorio y Equihua 2007; Salinas-Moreno et al. 2010; Raffa et al. 2015).

Ya que los escarabajos descortezadores compiten con los humanos por recursos o productos derivados de las plantas, son considerados como plagas forestales o agrícolas que causan importantes pérdidas económicas y que requieren intervenciones de manejo (Raffa et al. 2015). Este es el caso de especies de Dendroctonus, Scolytus, Ips y Phloeosinus, que son capaces de matar árboles sanos en los bosques. Otros géneros, como Gnathotrichus, Xyleborus y Xylosandrus, causan daños a la madera influyendo de manera significativa en su calidad (Equihua y Burgos 2002).

Actualmente existe preocupación por el efecto de los brotes de insectos descortezadores que generan la mortalidad masiva de árboles, lo cual podría incrementar como consecuencia del cambio climático global, especialmente en los bosques templados (Evans 2016). El aumento de la temperatura y los eventos de sequía influyen en el estado fisiológico de los árboles y en su capacidad para resistir el ataque de insectos (Allen et al. 2010), cuyas poblaciones y su capacidad de infestación aumentan (Del-Val y Sáenz-Romero 2017).

Considerando el papel ecológico y económico de los escarabajos descortezadores, el conocimiento de su ecología y distribución es un tema relevante para la conservación de los bosques y el manejo forestal sustentable (Burgos-Solorio y Equihua 2007), especialmente en el contexto del cambio climático global (Evans 2016). En este trabajo se reportan las especies de Scolytinae y Platypodinae, así como otras especies asociadas de las familias Histeridae, Cleridae y Trogossitidae que se comportan como depredadores de escarabajos de la corteza (Reséndiz-Martínez et al. 2016, Burhe 2017, Rubio-Ugalde et al. 2017, Wegensteiner et al. 2015), que fueron registradas durante un ciclo anual en un bosque mixto de pino-latifoliadas. El estudio se realizó como parte de una evaluación de causas de mortalidad de árboles en un área natural protegida, la Reserva de la Biosfera Sierra de Manantlán, Jalisco, México.

\section{MATERIAL Y MÉTODOS \\ Área de estudio}

Este trabajo se realizó en la Estación Científica Las Joyas (ECLJ), un sitio dedicado a la investigación ecológica de largo plazo y la educación, ubicado en una de las zonas núcleo de la Reserva de la Biosfera Sierra de Manantlán, en el estado de Jalisco, México (Santana et al. 2004). La Sierra de Manantlán es un macizo montañoso que forma parte del extremo noroccidental de la Sierra Madre del Sur, caracterizado por su alta diversidad biológica y un paisaje complejo, en el cual se encuentran desde selvas de zonas cálidas subhúmedas hasta bosques de coníferas y latifoliadas de zonas templado húmedas (INE 2000).

La ECLJ tiene una extensión de 1250 ha y se localiza entre los $19^{\circ} 34^{\prime} 14^{\prime \prime}-19^{\circ} 37^{\prime} 30^{\prime \prime}$ de latitud norte y $104^{\circ} 14^{\prime} 49^{\prime \prime}-104^{\circ} 18^{\prime} 16^{\prime \prime}$ de longitud oeste, en los límites de los municipios de Autlán de Navarro y Cuautitlán (Fig. 1). Presenta un gradiente elevacional de 1560 a $2242 \mathrm{~m}$ y un relieve montañoso con barrancas y hondonadas o "joyas" que dan su nombre al lugar. El clima es templado (temperatura media anual de $15.5^{\circ} \pm 0.2^{\circ} \mathrm{C}$ ), húmedo (cociente evapotranspiración potencial / precipitación anual de 0.5 0.6 ), con régimen de lluvias en verano (precipitación media anual $1860 \pm 94 \mathrm{~mm}$ ) y presenta condiciones de transición hacia clima semicálido subhúmedo en elevaciones menores a los 1600 m (Jardel-Peláez et al. 2004a).

La vegetación predominante en el $70 \%$ de la superficie de la ECLJ corresponde a bosques de pino con mezcla de encinos y otras latifoliadas; en las hondonadas y barrancas se encuentra bosque mesófilo de montaña (18\% de la superficie) y parte del área (12\%) corresponde a matorrales y herbazales secundarios, establecidos en sitios que en el pasado fueron desmontados para la agricultura (Jardel-Peláez et al. 2004b). En sitios con suelos profundos del orden de los Alfisoles, donde no han ocurrido incendios forestales por más de 20-40 años, se encuentran rodales mixtos dominados por Pinus douglasiana Martínez con mezcla de $P$. herrerae Martínez y P. oocarpa Schiede ex Schltdl. y latifoliadas como Carpinus tropicalis, Cornus disciflora, Clethra vicentina, Ilex brandegeana, Magnolia iltisiana, Myrsine juergensenii, Quercus calophylla, Symplocos citrea, Styrax ramirezii y Zinowiewia concinna, entre las más comunes, que forman un subdosel denso. Estudios realizados en el área indican que estos rodales representan una etapa de la sucesión ecológica que conduce al establecimiento del bosque mesófilo de montaña cuando se excluyen el fuego y el apacentamiento de ganado (Jardel-Peláez et al. 2004a). La mortalidad de árboles de pino causada por insectos descortezadores combinada con otros factores como competencia, envejecimiento, caída de árboles durante tormentas o el efecto de sequías, podrían estar influyendo en el proceso de reemplazo sucesional de los pinos por latifoliadas (Jardel-Peláez 2008).

\section{Establecimiento de sitios y registro de datos en campo}

En recorridos de campo en los bosques de la ECLJ y sus alrededores se eligieron dos rodales independientes de bosque mixto de pino-latifoliadas (Fig. 1), con condiciones similares de estructura y composición de especies arbóreas, elevación (entre 1880 y 1980 m), clima (templado húmedo), geomorfología (laderas de montaña medianamente diseccionadas con pendientes moderadas a fuertes) y tipo de suelo (Alfisol), partiendo de información de estudios previos (Jardel-Peláez et al. 2004a). Ambos rodales han tenido 


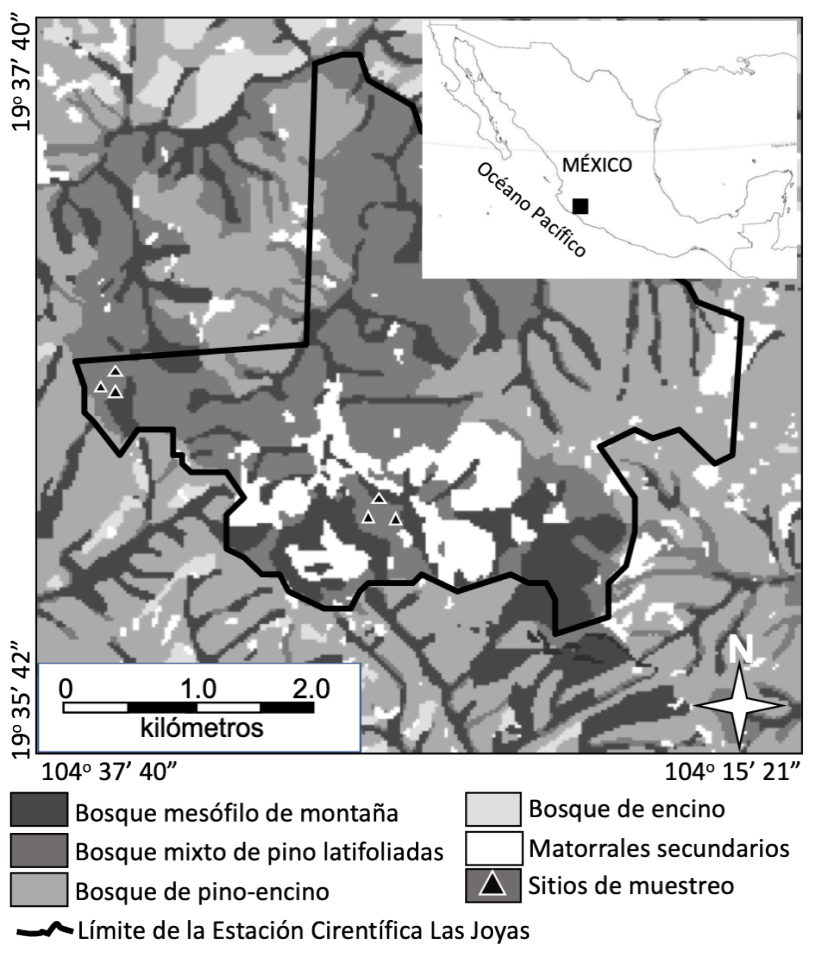

Figura 1. Localización y cobertura vegetal de la Estación Científica Las Joyas, Reserva de la Biosfera Sierra de Manantlán, Jalisco; los números indican la ubicación de los sitios de muestreo (rodales 1 у 2).

las mismas condiciones de manejo: fueron explotados para la producción de madera en 1959, tuvieron un incendio superficial en 1983 y desde 1987 se encuentran protegidos con exclusión de corta de madera, apacentamiento de ganado y supresión del fuego (Jardel-Peláez 2008). Estos rodales son conocidos localmente como Charco de los Perros y Cuchilla de la Tuna (de aquí en adelante rodal 1 y rodal 2 , respectivamente) y en los dos se encuentran árboles vivos y muertos con evidencia de ataque de insectos descortezadores.

En cada rodal se establecieron 3 sitios de muestreo, separados entre sí 300 metros aproximadamente y en cada uno se seleccionaron entre 2 y 4 árboles de cada una de las siguientes categorías: vivos sanos (sin evidencia de infestación por descortezadores), vivos infestados (con grumos de resina, agujeros de entrada o salida y aserrín en la corteza) y muertos en pie. En total fueron seleccionados 46 árboles ( 23 por rodal) y en cada uno de ellos se colocaron sobre el tronco, a una altura de $1.5 \mathrm{~m}$, dos tipos de trampas: de pegamento (trampas engomadas Trapper Max TM, de Laboratorios Bell Latam) y recipientes de plástico cebados con alcohol al 70\% como atrayente. Las trampas se colocaron y se dejaron durante una semana, para luego recolectar los insectos atrapados. El procedimiento se repitió mensualmente cubriendo un ciclo anual de julio de 2018 a agosto de 2019 . Los escarabajos de la corteza se determinaron como morfoespecies en el campo, contando el número de individuos capturados por trampa. Se guardaron en frascos con alcohol al 70\% para luego trasladarlos al laboratorio donde se montaron, etiquetaron y fotografiaron.

\section{Identificación de las especies y análisis de datos}

La determinación a nivel de especie o subfamilia, se realizó de manera preliminar en el Laboratorio de Zoología del Departamento de Ecología y Recursos Naturales de la Universidad de Guadalajara. Las determinaciones fueron completadas y corroboradas por el Dr. Armando Equihua Martínez en el Laboratorio de Entomología Forestal del Colegio de Postgraduados. Ejemplares de referencia fueron depositados en los dos laboratorios citados. La determinación de las especies se basó en los trabajos de Wood (1982), Díaz Ramos (2016), Armendariz-Toledano et al. (2018) y Atkinson (2019).

La abundancia total de individuos capturados por rodal en ambos tipos de trampas durante el ciclo anual, fue comparada gráficamente para los siguientes grupos de especies: descortezadores del género Dendroctonus, escarabajos ambrosiales y otros Curculionidae (Scolytinae y Platypodinae; incluyó a Hylurgops, Pseudips y Pityophthorus) y escarabajos de las familias Cleridae, Histeridae y Trogossitidae. Adicionalmente se hicieron pruebas de $t$ de Student (Zar 1999), para evaluar las diferencias en el número de capturas por grupo de especies entre las trampas de alcohol y las de pegamento.

\section{Especies registradas}

\section{RESULTADOS Y DISCUSIÓN}

De un total de 2470 colectas obtenidas durante un ciclo anual de muestreo, 1745 correspondieron a la familia Curculionidae, determinándose 17 especies de las cuales 15 corresponden a nueve géneros de la subfamilia Scolytinae y dos a un género de la subfamilia Platypodinae (Tabla 1). Se registraron adicionalmente dos especies de Trypanaeus y una de Platysoma (Histeridae), dos de Enoclerus y una de Cymatodera (Cleridae) y Temnoscheila virescens (Trogossitidae).

Diez de las especies de Curculionidae identificadas en este estudio representan nuevos registros para el estado de Jalisco: Amphicranus rameus Wood, 1967, A. melanuros Blandford, 1904, Corthylus comatus Blandford, 1904, C. fuscus Blandford, 1904, Gnathotrichus sulcatus LeConte, 1868, Hylurgops incomptus Blandford, 1896, Monarthrum laterale Eichhoff, 1859, Pseudips mexicanus Hopkins, 1905 (subfamilia Scolytinae) y Euplatypus longius Wood, 1966 y E. pini Hopkins, 1905 (subfamilia Platypodinae), de acuerdo con lo consignado en otros trabajos previos (Burgos-Solorio y Equihua 2007, Atkinson 2019).

\section{Hábitos alimenticios e importancia forestal}

De las especies de Scolytinae registradas, seis se alimentan del floema y pueden causar la muerte de árboles (Tabla 1). Sólo Dendroctonus mexicanus es conside- 
rada como una plaga forestal importante, mientras que $D$. approximatus y D. valens, al igual que Hylurgops incomptus, Pseudips mexicanus y Pityophthorus ca. bassetti, son considerados como insectos de importancia secundaria, ya que estas especies aceleran el proceso de descomposición y degradación de la celulosa, permitiendo el ingreso de otros organismos como hongos o bacterias a la madera mediante sus actividades reproductivas o de alimentación, asociándose a otras especies de insectos primarios (Cibrián et al. 1995). Las otras especies son barrenadores xilomicetófagos, de las cuales nueve son escolítinos de los géneros Amphicranus, Corthylus, Gnathotrichus, Monarthrum y Xyleborus y dos son platipodinos del género Euplatypus; estas especies juegan un papel importante en la descomposición de la madera de árboles muertos y pueden causar daño económico en la madera almacenada, especialmente Xyleborus ferrugineus (Equihua y Burgos 2002); esta especie infesta trocería húmeda apilada en campo o en aserraderos (Cibrián et al. 1995).

Las especies capturadas de las familias Histeridae, Cleridae y Trogossitidae se comportan como depredadoras de larvas o adultos de los escarabajos de la corteza (Reséndiz-Martínez et al. 2016, Burke 2017, Rubio-Ugalde et al. 2017) y pueden jugar un papel importante en la regulación de sus poblaciones (Wegensteiner et al. 2015).

\section{Distribución geográfica}

Respecto a la distribución geográfica (Tabla 1), se encontró una mezcla de especies de géneros de origen neotropical y neártico, como podría esperarse por la ubicación del área de estudio, en la Zona de Transición Mexicana (Halffter 2017). De acuerdo con el trabajo de Hulcr et al. (2015), de las 15 especies registradas de escolítidos, ocho son de géneros de distribución predominantemente neotropical y seis neártica; respecto a Pityophthorus ca. bassetti, esta pertenece a un género de amplia distribución en el cual la mayoría de las especies son neárticas y neotropicales, pero su identificación no es definitiva y por lo tanto no se conoce con exactitud su distribución.

Las especies de Dendroctonus (D. approximatus, D. mexicanus, y $D$. valens) presentan distribuciones geográficas amplias y se encuentran en la mayor parte de los sistemas montañosos de México (Salinas-Moreno et al. 2010). Corthylus detrimentosus se ha registrado en Durango, Guerrero, Jalisco, Estado de México, Michoacán y Morelos; Monarthrum conversum en los estados de Guerrero, Jalisco, Michoacán, Nayarit y Oaxaca (Atkinson 2019). Xyleborus ferrugineus es una especie de amplia distribución en México (Equihua y Burgos 2002).

Aunque varias de las especies encontradas de la subfamilia Scolytinae tienen una amplia distribución geográfica, no habían sido registradas previamente para el estado de Jalisco, siendo el caso de Amphicranus rameus, con una distribución conocida de Durango, Estado de México y Michoacán; A. melanurus de Panamá y Costa Rica, sólo conocida en México de Puebla; Corthylus comatus del centro de
México hasta Panamá y C. fuscus del centro de México a Guatemala y El Salvador; Gnathotrichus sulcatus desde El Salvador y Honduras hasta Canadá; Hylurgops incomptus del Suroeste de los Estados Unidos hasta Guatemala; $M o-$ narthrum laterale desde Brasil y Venezuela a Centroamérica y en México sólo se había registrado para Michoacán, Morelos, Oaxaca, Puebla y Veracruz; Pseudips mexicanus es de amplia distribución en las montañas de México. $P i$ tyophthorus ca. bassetti ha sido reportada para Jalisco en las localidades de Mascota y Tapalpa (Díaz Ramos 2016), presentando pocos individuos al igual que en el presente estudio y siendo sus hospederos Pinus donglasiana y Pinus devoniana, mientras que la especie cercana, $P$. bassetti, que se distribuye en Canadá y Estados Unidos, sólo se ha registrado en México en Picea chihuahuana en el estado de Chihuahua (Atkinson 2019).

Las dos especies encontradas de la subfamilia Platypodinae no se conocían para el estado de Jalisco. Trabajos anteriores registraron Euplatypus pini en Guatemala, Honduras, Nicaragua, Arizona, Nuevo México y en México en Chiapas, Durango, Hidalgo, Estado de México, Michoacán, Morelos, Nuevo León, Puebla, San Luís Potosí, Tlaxcala y Veracruz; E. longius se había reportado para Guatemala y Honduras y en México en Guerrero, Estado de México y Morelos y sus hospederos son latifoliadas como Clethra y Fraxinus (Atkinson 2019).

En otras partes de Jalisco como el Bosque La Primavera, se tienen registros de los géneros Hylurgops, Xyleborus, Gnathotrichus y Pityophthorus (Rodríguez-Rivas et al. 2018), también encontrados en la ECLJ, así como la especie Xyleborus ferrugineus. En Chamela, en selva baja caducifolia, Equihua y Atkinson (1986) registraron los géneros Corthylus y Pityophthorus así como la especie Xyleborus ferrugineus, también presente en la ECLJ.

Varias de las especies de escarabajos de la corteza registradas en la ECLJ se han encontrado también en bosques templados del norte del estado de Morelos (Atkinson et al. 1986); se comparten las tres especies de Dendroctonus (D. valens, D. approximatus y D. mexicanus), las tres de Corthylus (C. fuscus, C. comatus y C. detrimentosus), Hylurgops incomptus, Monarthrum laterale, Euplatypus longius, E. pini, Pseudips mexicanus, así como los géneros Xyleborus, Pityophthorus y Amphicranus, aunque estos con especies diferentes. Esto puede deberse a la similitud de condiciones de clima templado y vegetación de bosque de pino-encino y latifoliadas; por ejemplo la mayoría de las especies del género Pityophthorus se asocian a comunidades templadas y frías como bosque de pino y pino-encino (Burgos-Solorio y Equihua 2007).

La riqueza de especies reportada por Atkinson et al. (1986) para los bosques del norte de Morelos (130 especies), así como la registrada en Chamela (99 especies) por Equihua y Atkinson (1986), es mucho mayor a la reportada en la ECLJ (17 especies). Esta diferencia se debe a que en el presente estudio solamente se colectaron los individuos atraídos a las trampas en dos localidades, sin buscar en 
plantas hospederas diferentes a los pinos, como las latifoliadas, en las cuales se sabe que pueden habitar varias especies de Scolytinae (Atkinson et al. 1986). Esto indica la necesidad de profundizar en el estudio de la comunidad de escarabajos de la corteza de la ECLJ, que potencialmente puede ser más rica en especies.

\section{Comparación entre los tipos de trampas}

La comparación entre las medias del número de capturas por tipo de trampas (Fig. 2) se realizó con el total de trampas utilizadas (43 de cada tipo) y sólo mostró diferencias estadísticamente significativas para los escarabajos ambrosiales $(\mathrm{t}=3.53, \mathrm{p}=0.001)$, siendo más efectivas las trampas de alcohol que las de pegamento (media del número de capturas $19.7 \pm 2.3$ y $8.8 \pm 2$ respectivamente). Para el género Dendroctonus fue mayor la captura en las trampas de alcohol (4.9 \pm 1.1$)$ que en las de pegamento $(2.4 \pm 0.6)$, pero la diferencia no fue estadísticamente significativa $(\mathrm{t}=$ $-1.95, \mathrm{p}=0.055)$. En el caso de Histeridae no hubo diferencias entre los dos tipos de trampas (media $7.0 \pm 1.1$ y $7.2 \pm$ 2.2 en alcohol y pegamento respectivamente; $\mathrm{t}=-0.11, \mathrm{p}=$ 0.916) (Fig. 2). La mayor eficiencia en la captura con las trampas de alcohol, podría atribuirse a que la sustancia que se utiliza como atrayente es más similar a las encontradas en la resina y corteza de los árboles que utilizan como hospederos los descortezadores (Burgos-Solorio 1998, Krokene 2015).

\section{Diferencias entre los rodales}

Los rodales mostraron diferencia en el número total de individuos colectados en los dos tipos de trampas; la abundancia fue mayor en el rodal 2 que en el rodal 1 para todos los grupos de insectos (Fig. 3). El grupo más abundante en ambos rodales fue el de los escarabajos ambrosiales, seguido de Histeridae y Dendroctonus; las familias Cleridae y Trogossitidae fueron las que tuvieron las menores abundancias. De acuerdo con las observaciones de campo y considerando las categorías propuestas de árboles infestados que reflejan las diferentes etapas del ataque de los descortezadores (Billings et al. 2019), la diferencia en el número de capturas se atribuye a que el rodal 1 se encontraba en una transición entre las etapas dos (pinos con crías de Dendroctonus) y tres (pinos muertos y abandonados), mientras que el rodal 2 se encontraba en la fase uno (pinos con ataques recientes) por lo que pudiera presentar más especies de insectos. En cuanto a la riqueza de especies, en el rodal 1 se presentaron ocho géneros y 13 especies contra diez géneros con 17 especies en el rodal 2 (Tabla 1). En el caso de ambrosiales solamente Gnathotrichus sulcatus, Euplatypus pini y posiblemente Xyleborus ferrugineus están asociados a pinos; las otras especies han sido registradas en latifoliadas como hospederos (Atkinson 2019).

Las diez especies encontradas como nuevos registros para el estado de Jalisco en esta investigación, aumentan a un total de 206 especies según lo reportado previamente por Burgos-Solorio y Equihua (2007) y Díaz Ramos

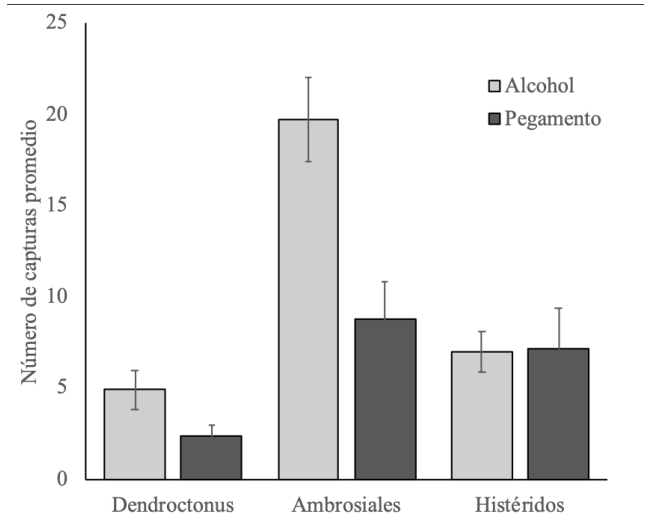

Figura 2. Número de capturas promedio (con barras de error estándar) de Dendroctonus, escarabajos ambrosiales y otros curculiónidos e histéridos en los dos tipos de trampas.

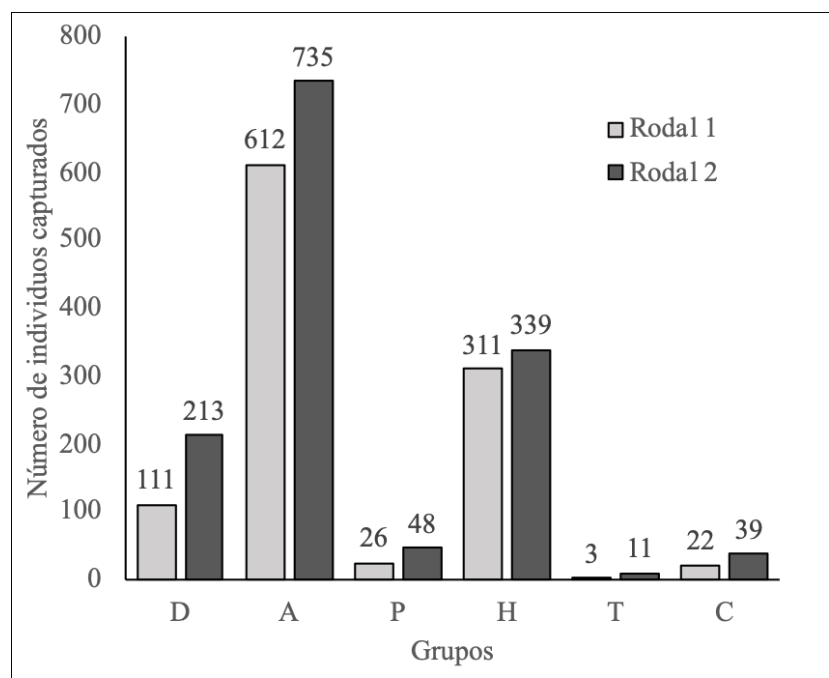

Figura 3. Número de capturas totales de individuos por rodal de los grupos de escarabajos: D, Dendroctonus; A, escarabajos ambrosiales y otros Curculionidae; P, Platypodinae; H, Histeridae; T, Trogossitidae; C, Cleridae.

(2016). Se trata de especies que pueden considerarse de amplia distribución, lo cual indica la necesidad de ampliar los estudios en diferentes localidades, para contar con mejor información sobre la distribución de las especies de escarabajos de la corteza, considerando su importancia ecológica y económica. El estudio de la composición de las comunidades de escarabajos de la corteza, de la dinámica de la abundancia y distribución espacial y temporal de sus poblaciones, y de las interacciones con sus enemigos naturales es indispensable para entender mejor su papel en los procesos ecológicos en los bosques y fundamentar el diseño de mejores prácticas de manejo de los brotes de insectos que causan pérdidas económicas.

\section{AGRADECIMIENTOS}

Este estudio formó parte de la tesis de Maestría en Ciencias en Manejo de Recursos Naturales que la primera autora realizó en el Centro Universitario de la Costa Sur de la Univer- 
sidad de Guadalajara, contando con la beca 854833/629668 otorgada por el Consejo Nacional de Ciencia y Tecnología (CONACYT). Se agradece a Erminio Quiñonez Ramos, Montserrat Barragán Vicente, M. Goretti Cantón Muñiz y Georgina Zarco Velazco y al personal de la Estación Científica Las Joyas por el apoyo durante el trabajo de campo, así como a los dos revisores anónimos cuyos comentarios ayudaron a mejorar este trabajo.

\section{LITERATURA CITADA}

Allen, C.D., A.K. Macalady, H. Chenchouni, D. Bachelet and N. Mcdowell. 2010. A global overview of drought and heat-induced tree mortality reveals emerging climate change risks for forests. Forest Ecology and Management, 259(4): 660-684.

Armendáriz-Toledano, F., G. Zúñiga, L.J. García-Román, O. Valerio-Mendoza y P.G. García-Navarrete (Eds). 2018. Guía ilustrada para identificar a las especies del género Dendroctonus presentes en México y Centroamérica. Instituto Politécnico Nacional. Ciudad de México, México.

Atkinson, T.H., C.E. Saucedo, F.E. Martínez y A. Burgos Solorio.1986. Coleópteros Scolytidae y Platypodidae asociados con las comunidades de clima templado y frío en el estado de Morelos, México. Acta Zoológica Mexicana, (17): 1-87.

Atkinson, T.H. 2017. Familia Curculionidae: Scolytinae y Platypodinae. (pp. 269-278) En: Cibrián, T.D. (Ed.). Fundamentos de Entomología Forestal. Universidad Autónoma de Chapingo, Texcoco, Estado de México.

Atkinson, T.H. 2019. Bark and ambrosia Beetles. https:// www.barkbeetles.info/regional_chklist_expanded. php?user_geo $=$ calc_mexico\%3D1\&user_geo_title $=$ Mexico\%20(country). Fecha de consulta: 19 de abril de 2019.

Billings, R.F., H.A. Pase III, L. J.E. Flores y Texas Forest Service. 2018. Los escarabajos descortezadores del pino, con énfasis en Dendroctonus frontalis: Guía de campo para la inspección terrestre. Disponible en: https://www.barkbeetles.org/spb/indice3a.html. Fecha de consulta: 3 de Agosto de 2019.

Burke, A.F. 2017. Systematics and biogeography of the subfamily Tillinae (Coleoptera: Cleridae) in the New World. Doctoral Thesis. Kansas State University, Manhattan, Kansas.

Burgos-Solorio, A. 1998. Escarabajos barrenadores Platypodidae y Scolytidae (Coleoptera) atraídos a trampas NTP-80 de la cañada de los alrededores de San José de los Laureles, Tlayacapan, Morelos, México. Dugesiana, 5(2): 29-34.

Burgos-Solorio, A. y A. Equihua. 2007. Platypodidae y Scolytidae (Coleoptera) de Jalisco, México. Dugesiana, 14(2): 59-82.

Cibrián, T.D., J.T. Méndez, R. Campos, H.O. Yates III y J. Flores (Eds.). 1995. Insectos Forestales de México / Forest Insects of Mexico. (COFAN/NAFC). Primera
Edición. Universidad Autónoma Chapingo. Texcoco, Estado de México.

Del-Val, E. y C. Sáenz-Romero. 2017. Insectos descortezadores (Coleoptera: Curculionidae) y cambio climático: problemática actual y perspectivas en los bosques templados. Revista Especializada en Ciencias Químico-Biológicas, 20(2): 53-60.

Díaz Ramos, S.G. 2016. El género Pityophthorus spp. (Coleoptera: Curculionidae: Scolytinae) en bosques templados de pino en Jalisco. Tesis de Maestría en Ciencias, Colegio de Postgraduados, Montecillo, Estado de México.

Equihua, M.A. and S.A. Burgos. 2002. Scolytidae. (pp. 539-557). En: B.J. Llorente and J.J. Morrone (Eds.). Biodiversidad, taxonomía y biogeografía de artrópodos de México: Hacia una síntesis de su conocimiento. Vol. III.CONABIO-IBUNAM. México.

Equihua, M.A., and T.H. Atkinson. 1986. Annotated checklist of the bark and ambrosia beetles (Coleoptera: Scolytidae and Platypodidae) associated with tropical deciduous forest in Chamela, Jalisco, Mexico. Florida Entomol, (69): 619-635.

Evans, A.M. 2016. Invasive plants, insects, and diseases in the forests of the Anthropocene. (pp. 33-42). In: Sample, V.A., R.P. Bixler y C. Miller (Eds.) Forest conservation in the anthropocene. University Press of Colorado, Boulder, United States of America.

Halffter, G. 2017. La zona de transición mexicana y la megadiversidad de México: del marco histórico a la riqueza actual. Dugesiana, 24(2): 77-89.

Hulcr, J., T.H. Atkinson., A.I. Cognato, B.H. Jordal and D.D. Mckenna. 2015. Morphology, taxonomy, and phylogenetic of bark beetles. (pp. 41-64). In: Vega F.E. and R.W. Hofstetter (Eds.). Bark beetles biology and ecology of native and invasive species. Elsevier. Academic Press, United States of America.

Instituto Nacional de Ecología (INE). 2000. Programa de manejo de la Reserva de la Biosfera Sierra de Manantlán. Secretaria de Medio Ambiente, Recursos Naturales y Pesca/Instituto Nacional de Ecología. México.

Jardel-Peláez, E.J., E. Ezcurra, R. Cuevas-Guzmán y P. Cruz C. 2004 a. Vegetación y patrones del paisaje. (pp. 67-115). En: R. Cuevas-Guzmán y E.J. Jardel (Eds.). Flora y vegetación de la Estación Científica Las Joyas. Universidad de Guadalajara, Autlán de Navarro, Jalisco. Jardel-Peláez, E.J., A.L. Santiago-Pérez, C. Cortés M. y F. Castillo-Navarro. 2004b. Sucesión y dinámica de rodales. (pp. 179-203). En: R. Cuevas-Guzmán y E.J. Jardel-Peláez (Eds.). Flora y vegetación de la Estación Cientifica Las Joyas. Universidad de Guadalajara, Autlán de Navarro, Jalisco.

Jardel-Peláez, E.J. 2008. Sucesión ecológica y restauración de bosques tropicales de montaña en la Estación Científica Las Joyas, México. En: González- Espinoza, M., J.M. Rey-Benayas y N. Ramírez-Marcial (Eds.). Restauración de bosques en América Latina. Fundación 
Internacional para la Restauración de Ecosistemas y Mundi-Prensa. México.

Kovarik, P.W. and M.S. Caterino. 2016. Histeridae. (pp. 281-314). In: Beutel, R.G. and Leschen, R.A.B. (Eds.). Handbook of Zoology, Arthropoda: Insecta. Coleoptera, Beetles. Vol. 1: Morphology and Systematics (Archostemata, Adephaga, Myxophaga, Polyphaga partim). Walter de Gruyter, Berlin.

Krokene P. 2015. Conifer defense and resistance to bark beetles. (pp.177-202). In: Vega F.E. and R.W. Hofstetter (Eds.). Bark beetles biology and ecology of native and invasive species. Elsevier. Academic Press, United States of America.

Raffa, K.F., J.C. Gregoire, and S.B. Lindgren. 2015. Natural history and ecology of bark beetles. (pp. 1-28). In: Vega F.E. and R.W. Hofstetter (Eds.). Bark beetles biology and ecology of native and invasive species. Elsevier. Academic Press, United States of America.

Reséndiz-Martínez, J.F., B. Torres-Huerta, V. López-Gómez, A. Gijón-Hernández y G. Sánchez-Martínez. 2016. Enemigos naturales de Dendroctonus frontalis Zimmerman, 1868 y Dendroctonus mexicanus Hopkins, 1915 (Coleoptera: Scolytinae), capturados mediante semioquímicos en la Reserva de la Biosfera Sierra Gorda de Querétaro. Entomología Mexicana, (3): 626-632.

Rodríguez-Rivas, A., S.G. Díaz-Ramos, H.J. Contreras-Quiñones, L. Barrientos-Ramírez T. Escoto García y A. Equihua-Martínez. 2018. Registro de escarabajos descortezadores (Curculionidae: Scolytinae) en el Bosque La Primavera, Jalisco. Revista Mexicana de Ciencias Forestales, 9(48): 135-149.

Recibido: 17 de diciembre 2019

Aceptado: 18 de abril 2020
Rubio-Ugalde, D.J., V.H. Cambrón-Sandoval y S. Vergara-Pineda. 2017. Coleópteros depredadores asociados al sistema de monitoreo de escarabajos descortezadores (Curculionidae: Scolytinae) en el Tepozán, Arroyo Seco, Querétaro. Entomología Mexicana, (4): 186-191.

Salinas-Moreno, Y., C.F. Vargas-Mendoza, G. Zúñiga, J. Víctor, A. Ager y J.L. Hayes (Eds.). 2010. Atlas de distribución geográfica de los descortezadores del género Dendroctonus (Curculionidae: Scolytinae) en México. Instituto Politécnico Nacional, México, D.F.

Santana, C.E., E.J. Jardel-Peláez, V.F. Hernández, R. Cuevas-Guzmán, D. Partida L., L.I. Iñiguez D., y L.E. Rivera-Cervantes. 2004. Investigación y educación en un área natural protegida. (pp. 9-35). En: R. Cuevas-Guzmán y E.J. Jardel-Peláez (Eds.). Flora y vegetación de la Estación Científica Las Joyas. Universidad de Guadalajara, Autlán de Navarro, Jalisco.

Wegensteiner, R., B. Wermelinger and M. Herrmann. 2015. Natural enemies of bark beetles: predators, parasitoids, pathogens, and nematodes. (pp. 247-289). In: Vega F.E. and R.W. Hofstetter (Eds.).Bark beetles biology and ecology of native and invasive species. Elsevier. Academic Press, United States of America.

Wood, S.L. 1982.The bark and ambrosia beetles of North and Central America (Coleoptera: Scolytidae), a taxonomic monograph. Great Basin Naturalis Memoirs, (6): 33-44.

Zar, J.H. 1999. Biostatistical Analysis. Prentice Hall, Nueva York. 
Tabla 1. Lista de especies de coleópteros colectadas en la corteza de pinos en el bosque mixto de pino-latifoliadas de la Estación Científica Las Joyas, de julio de 2018 a agosto de 2019. Se indican sus hábitos alimenticios, distribución biogeográfica a nivel de género y su presencia en los dos rodales estudiados. Las especies marcadas con asterisco son nuevos registros para el estado de Jalisco.

\begin{tabular}{|c|c|c|c|c|}
\hline Familias y especies & $\begin{array}{l}\text { Hábitos alimenti- } \\
\text { cios }\end{array}$ & Distribución ${ }^{(2)}$ & Rodal 1 & Rodal 2 \\
\hline \multicolumn{5}{|l|}{ Familia Curculionidae } \\
\hline \multicolumn{5}{|l|}{ Subfamilia Scolytinae } \\
\hline Amphicranus melanurus Blandford, 1904* & Xilomicetófago & Neotropical & & $\mathrm{X}$ \\
\hline Amphicranus rameus Wood, 1967* & Xilomicetófago & Neotropical & $\mathrm{X}$ & $\mathrm{X}$ \\
\hline Corthylus comatus Blandford, 1904* & Xilomicetófago & Neotropical & $\mathrm{X}$ & $\mathrm{X}$ \\
\hline Corthylus detrimentosus Schedl, 1940 & Xilomicetófago & Neotropical & & $\mathrm{X}$ \\
\hline Corthylus fuscus Blandford, 1904* & Xilomicetófago & Neotropical & $\mathrm{X}$ & $\mathrm{X}$ \\
\hline Dendroctonus approximatus Dietz, 1890 & Fleófago & Neártico & $\mathrm{X}$ & $\mathrm{X}$ \\
\hline Dendroctonus mexicanus Hopkins, 1905 & Fleófago & Neártico & $\mathrm{X}$ & $\mathrm{X}$ \\
\hline Dendroctonus valens LeConte, 1859 & Fleófago & Neártico & $\mathrm{X}$ & $\mathrm{X}$ \\
\hline Gnathotrichus sulcatus (LeConte, 1868)* & Xilomicetófago & Neártico & $\mathrm{X}$ & $\mathrm{X}$ \\
\hline Hylurgops incomptus (Blandford, 1896)* & Fleófago & Neártico & $\mathrm{X}$ & $\mathrm{X}$ \\
\hline Monarthrum conversum Wood, 1974 & Xilomicetófago & Neotropical & $\mathrm{X}$ & $\mathrm{X}$ \\
\hline Monarthrum laterale (Eichhoff, 1859)* & Xilomicetófago & Neotropical & $\mathrm{X}$ & $\mathrm{X}$ \\
\hline Pityophthorus ca. bassetti Blackman, 1920 & Fleófago & Neártico & & $\mathrm{X}$ \\
\hline Pseudips mexicanus (Hopkins, 1905)* & Fleófago & Neártico & $\mathrm{X}$ & $\mathrm{X}$ \\
\hline Xyleborus ferrugineus (Fabricius, 1801). & Xilomicetófago & Neotropical & & $\mathrm{X}$ \\
\hline \multicolumn{5}{|l|}{ Subfamilia Platypodinae } \\
\hline Euplatypus longius (Wood, 1966)* & Xilomicetófago & Neotropical & $\mathrm{X}$ & $\mathrm{X}$ \\
\hline Euplatypus pini (Hopkins, 1905)* & Xilomicetófago & Neotropical & $\mathrm{X}$ & $\mathrm{X}$ \\
\hline \multicolumn{5}{|l|}{ Familia Histeridae } \\
\hline Platysoma sp. & Insectívoro & Cosmopolita & $\mathrm{X}$ & $\mathrm{X}$ \\
\hline Trypanaeus $\mathrm{sp} .1$ & Insectívoro & Neotropical & $\mathrm{X}$ & $\mathrm{X}$ \\
\hline Trypanaeus sp. 2 & Insectívoro & Neotropical & $\mathrm{X}$ & $\mathrm{X}$ \\
\hline \multicolumn{5}{|l|}{ Familia Cleridae } \\
\hline Enoclerus arachnodes (Klug, 1842) & Insectívoro & Neártico & $\mathrm{X}$ & $\mathrm{X}$ \\
\hline Enoclerus sp. & Insectívoro & Neártico & & $\mathrm{X}$ \\
\hline Cymatodera sp. & Insectívoro & Neártico & $\mathrm{X}$ & $\mathrm{X}$ \\
\hline \multicolumn{5}{|l|}{ Familia Trogossitidae } \\
\hline Temnoscheila virescens (Fabricius, 1801) & Insectívoro & Neártico & $\mathrm{X}$ & $\mathrm{X}$ \\
\hline
\end{tabular}

(1) Hábitos alimenticios de acuerdo con Atkinson (2017), Burgos-Solorio y Equihua (2007) y Atkinson et al. (1986).

(2) Distribución biogeográfica a nivel de género basada en Hulcr et al. (2015), Kovarik y Caterino (2016) y Burke 2017. 